\title{
STOCHASTIC ORDERS AND THE FROG MODEL
}

\author{
TOBIAS JOHNSON AND MATTHEW JUNGE
}

\begin{abstract}
The frog model starts with one active particle at the root of a graph and some number of dormant particles at all nonroot vertices. Active particles follow independent random paths, waking all inactive particles they encounter. We prove that certain frog model statistics are monotone in the initial configuration for two nonstandard stochastic dominance relations: the increasing concave and the probability generating function orders.

This extends many canonical theorems. We connect recurrence for random initial configurations to recurrence for deterministic configurations. Also, the limiting shape of activated sites on the integer lattice respects both of these orders. Other implications include monotonicity results on transience of the frog model where the number of frogs per vertex decays away from the origin, on survival of the frog model with death, and
\end{abstract} on the time to visit a given vertex in any frog model.

Le modèle de la grenouille se base sur un graphe contenant une particule active à sa racine et un certain nombre de particules dormantes sur ses autres sites. Les particules actives suivent des chemins aléatoires, et éveillent les particules inactives qu'elles rencontrent. Nous montrons que certaines statistiques des modèles de grenouilles sont monotones en leur configuration initiale pour deux relations d'ordre: l'ordre concave croissant et l'ordre des fonctions génératrices.

Ces résultats étendent de nombreux théorèmes canoniques. On établit un lien entre la récurrence de la configuration initiale et la récurrence de configurations déterministes. De plus, dans le graphe naturel des entiers, la forme limite des sites activés respecte ces deux relations d'ordre. D'autres conséquences concernent des résultats de monotonicité sur la transience du modèle de grenouille où le nombre de grenouilles par site diminue lorsque l'on s'éloigne de l'origine, sur la survie dans un modèle intégrant la mort, et sur les temps de visite a un site donné dans n'importe quel modèle de grenouille.

\section{INTRODUCTION}

Let $G$ be a countable collection of vertices, one of which we distinguish as the root and call $\varnothing$. A general frog model $(\eta, S)$ starts with one active particle at $\varnothing$ and $\eta(v)$ dormant particles at each $v \neq \varnothing$. The $i$ th particle at $v$ starting from its time of activation moves according to the path $S .(v, i)$, with $S_{0}(v, i)$ assumed equal to $v$. When an active particle visits a site containing dormant particles, all of the dormant particles activate. The particles move in discrete time, though this will be unimportant since most of the properties of the frog model we consider depend only on the particles' paths and not on the time they make their moves. The particles are traditionally called frogs, and we continue the zoomorphism. Typically, $G$ is a graph, the frog paths $(S \cdot(v, i))_{v \in G, i \geq 1}$ are independent random walks, the

Date: April 6, 2017.

2010 Mathematics Subject Classification. 60K35, 60J80, 60J10.

Key words and phrases. Frog model, transience, recurrence, phase transition, stochastic orders, increasing concave order, probability generating function order.

The first author was partially supported by NSF grant DMS-1401479. 
frog counts $(\eta(v))_{v \in G}$ are either deterministic or i.i.d., and $(S .(v, i))_{v \in G, i \geq 1}$ and $(\eta(v))_{v \in G}$ are independent of each other. We will not belabor an example like the frog model with simple random walk paths on $\mathbb{Z}^{d}$ and i.i.d.-Poi $(\mu)$ frogs per vertex by stating that the frog paths are mutually independent, and that the frog counts and paths are independent.

Our main result is about two classes of frog model functionals we call icv and pgf statistics. The prime example is the number of visits to $\varnothing$ in the frog model $(\eta, S)$ over all time, which we denote $r(\eta, S)$. A realization of the frog model is called recurrent if $r(\eta, S)=\infty$ and transient otherwise. In TW99, the frog model with one sleeping frog per site and simple random walk paths is shown to be recurrent almost surely on $\mathbb{Z}^{d}$ for all $d$. This is further refined in Pop01, which exhibits a threshold in $\alpha$ at which a frog model with Bernoulli $\left(\alpha\|x\|^{-2}\right)$ frogs at each $x \in \mathbb{Z}^{d}$ switches from transience to recurrence. A similar phenomenon occurs when the walks have a bias in one direction: GS09] finds that on $\mathbb{Z}$, the model is recurrent if and only if the number of sleeping frogs per site has infinite logarithmic moment. A sufficient condition for recurrence in this setting on $\mathbb{Z}^{d}$ was given in [DP14] and improved on in KZ16. In our papers HJJ16b, HJJ16a, JJ16, we prove that the frog model with simple random walk paths on the infinite $d$-ary tree $\mathbb{T}_{d}$ switches from transient to recurrent either when the density of frogs increases with $d$ held fixed, or when $d$ decreases with the density held fixed.

Statement of main theorem. Our main result is a comparison theorem relating icv and pgf statistics of a frog model (see Definition 1) when we vary the distribution of the initial configuration $\eta$. Our original motivation was that while the most convenient setting in our experience has Poisson-distributed frog counts, the most basic questions assume a deterministic number of frogs per site. As an example, in HJJ16a we showed the existence of a recurrence phase on the $d$-ary tree with Poisson frogs per site for any $d \geq 2$. This left open the existence of a recurrence phase for initial conditions other than i.i.d. Poisson. For instance, for large enough $k$, is the frog model recurrent on the $d$-ary tree with $k$ frogs per site? With our previous tools, we could answer this question only for the case $d=2$ HJJ16b, but our comparison theorem tidily transfers the result from Poisson to deterministic initial conditions (see Corollary 6).

If the initial condition $\eta(v)$ is dominated by $\eta^{\prime}(v)$ in the usual stochastic order, then we can couple the corresponding frog models and deduce that $f(\eta, S)$ is dominated by $f\left(\eta^{\prime}, S\right)$ for any statistic $f$ that is increasing in $\eta$. This is not helpful for the problem described above, since we cannot relate a Poisson random variable to the constant $k$ in this stochastic order. Instead, our main theorem will show that if $\eta(v)$ is dominated by $\eta^{\prime}(v)$ in a weaker stochastic order, then $f(\eta, S)$ will be dominated by $f\left(\eta^{\prime}, S\right)$ for all $f$ in a certain class of statistics.

First, we define the two weaker stochastic orders we use (see Section 2 for further discussion). For two random variables $X$ and $Y$ taking values in $[0, \infty]$, we say that $X$ is dominated by $Y$ in the increasing concave order if $\mathbf{E} f(X) \leq \mathbf{E} f(Y)$ for all bounded increasing concave functions $f:[0, \infty) \rightarrow \mathbb{R}$, with $f(\infty)$ taken as $\lim _{x \rightarrow \infty} f(x)$. We denote this by $X \preceq_{\text {icv }} Y$. We say that $X$ is dominated by $Y$ in the probability generating function order if $\mathbf{E} t^{X} \geq \mathbf{E} t^{Y}$ for all $t \in(0,1)$, with $t^{\infty}$ interpreted as 0 , and we denote this by $X \preceq_{\text {pgf }} Y$. From now on, we abbreviate increasing concave order to icv order and probability generating function order to pgf order. Since $x \mapsto 1-t^{x}$ is a bounded increasing concave function, $X \preceq_{\text {icv }} Y$ implies $X \preceq_{\text {pgf }} Y$. The icv order has come up several times in discrete probability, most notably in first passage percolation vdBK93, Mar02. See also Zer98 for an application to random walk in a random potential. In these papers, the relation $\pi_{1} \preceq_{\text {icv }} \pi_{2}$ is referred 
to as $\pi_{2}$ being more variable than $\pi_{1}$. The only use of the pgf order that we know of in discrete probability is our own in [HJJ16b], though see [TRZ11, LT14 for some applications in signal processing and wireless networks under the name Laplace transform order.

Next, we define the classes of statistics covered by our main theorems. Roughly speaking, we call a function of the frog model an icv statistic if it increases when a frog is added to the model, but when two frogs are added at the same vertex it increases less than by the separate addition of each of them. The pgf statistics form a more restrictive class of frog model functionals that obey a higher order version of this property. As we will prove in Section 4, many natural frog model statistics fall into these classes, most notably the count $r(\eta, S)$ of visits to the root.

Before we give the definitions, we will need to introduce some notation. Let

$$
\{\eta(v), S .(v, i): v \in G, i \geq 1\}
$$

be a deterministic collection of frog counts and paths. For any path $P_{\bullet}$, let $\sigma_{P \cdot}(\eta, S)$ denote

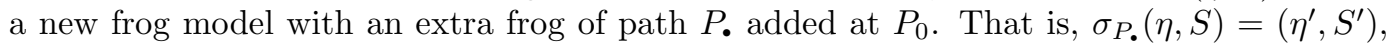
where $\eta^{\prime}$ is identical to $\eta$ except that $\eta^{\prime}\left(P_{0}\right)=\eta\left(P_{0}\right)+1$, and $S^{\prime}$ is identical to $S$ except that $S_{\bullet}^{\prime}\left(P_{0}, \eta\left(P_{0}\right)+1\right)=P_{\text {. }}$. For any frog model statistic $f(\eta, S)$, define

$$
\Delta_{P_{\mathbf{0}}} f(\eta, S)=f\left(\sigma_{P_{\mathbf{v}}}(\eta, S)\right)-f(\eta, S),
$$

the change in $f$ when a frog with path $P$. is added to the model.

Definition 1. Let $f$ be a functional of the frog model taking values in $[0, \infty]$. We call $f$ an icv statistic if the following conditions hold for all $(\eta, S)$ and all paths $P_{\bullet}^{1}, \ldots, P_{\bullet}^{m}$ starting at the same vertex:

(i) for $m=1,2$,

$$
(-1)^{m} \Delta_{P_{\bullet}^{1}} \cdots \Delta_{P_{\bullet}^{m}} f(\eta, S) \leq 0
$$

whenever all quantities in the expansion of the left hand side of the inequality are finite;

(ii) if $f(\eta, S)=\infty$, then $f\left(\sigma_{P_{0}^{1}}(\eta, S)\right)=\infty$;

(iii) if $f\left(\sigma_{P_{\bullet}^{1}} \sigma_{P_{\bullet}^{2}}(\eta, S)\right)=\infty$, then $f\left(\sigma_{P_{\bullet}^{i}}(\eta, S)\right)=\infty$ for either $i=1$ or $i=2$.

If $f$ satisfies conditions (ii) and (iii), and it satisfies condition (i) not just for $m=1,2$ but for all $m \geq 1$, then we call $f$ a pgf statistic. In either case, we call the statistic continuous if the condition

$$
\eta_{k}(v) \nearrow \eta(v) \text { as } k \rightarrow \infty \text { for all } v \in G
$$

implies that $f\left(\eta_{k}, S\right) \nearrow f(\eta, S)$ as $k \rightarrow \infty$.

The $m=1$ case of (i) is the condition that $f$ increases when a new frog is added. To make the $m=2$ case more transparent, we can restate it as

$$
f\left(\sigma_{P_{\bullet}^{1}} \sigma_{P_{\bullet}^{2}}(\eta, S)\right)-f\left(\sigma_{P_{\bullet}^{1}}(\eta, S)\right)-f\left(\sigma_{P_{\bullet}^{2}}(\eta, S)\right)+f(\eta, S) \leq 0 .
$$

Shifting terms around, we have the equivalent condition

$$
f\left(\sigma_{P_{\bullet}^{1}} \sigma_{P_{\bullet}^{2}}(\eta, S)\right)-f(\eta, S) \leq\left(f\left(\sigma_{P_{\bullet}^{1}}(\eta, S)\right)-f(\eta, S)\right)+\left(f\left(\sigma_{P_{\bullet}^{2}}(\eta, S)\right)-f(\eta, S)\right),
$$

which states that the gain to the statistic by adding two frogs at the same vertex is less than the combined gain of adding each frog separately, as we mentioned earlier.

Conditions (ii) and (iii) are essentially the conditions of being increasing and concave extended to apply when the statistic takes infinite values. We also mention that condition(iii) implies the apparently stronger condition that for any $(\eta, S)$ and any paths $P_{\bullet}^{1}, \ldots, P_{\bullet}^{m}$ 
starting at the same vertex, if $f\left(\sigma_{P_{\bullet}^{1}} \cdots \sigma_{P_{\bullet}^{m}}(\eta, S)\right)=\infty$, then $f\left(\sigma_{P_{\bullet}^{i}}(\eta, S)\right)=\infty$ for some $i$. Indeed, suppose that $f\left(\sigma_{P_{\bullet}^{1}} \cdots \sigma_{P_{\bullet}^{m}}(\eta, S)\right)=\infty$. Let $i_{1}, \ldots, i_{\ell}$ be any minimal set of indices such that $f\left(\sigma_{P_{\bullet}^{i_{1}}} \cdots \sigma_{P_{\bullet}^{i_{\ell}}}(\eta, S)\right)=\infty$. If $\ell \geq 2$, then with $\left(\eta^{\prime}, S^{\prime}\right)=\sigma_{P_{\bullet}^{i_{3}}} \cdots \sigma_{P_{\bullet}^{i_{\ell}}}(\eta, S)$, we have $f\left(\sigma_{P_{0}^{i_{1}}} \sigma_{P_{0}^{i_{2}}}\left(\eta^{\prime}, S^{\prime}\right)\right)=\infty$ even though $f\left(\sigma_{P_{0}^{i_{1}}}\left(\eta^{\prime}, S^{\prime}\right)\right)<\infty$ and $f\left(\sigma_{P_{0}^{i_{2}}}\left(\eta^{\prime}, S^{\prime}\right)\right)<\infty$. This is impossible by condition (iii). Hence $\ell=1$, proving the stronger condition.

Remark 2. The definition of icv and pgf statistics could be made in a more abstract setting. Suppose that $\mathcal{X}$ is the space of point process configurations on a space $S$. For any $f: \mathcal{X} \rightarrow$ $[0, \infty], \chi \in \mathcal{X}$, and $x \in S$, define $\Delta_{x} f(\chi)=f\left(\chi+\delta_{x}\right)-f(\chi)$. We could then call $f$ an icv statistic if for any $x_{1}, \ldots, x_{n} \in S$ and $n=1,2$,

$$
(-1)^{n} \Delta_{x_{1}} \cdots \Delta_{x_{n}} f(\chi) \leq 0
$$

and if statements analogous to condition(ii) and(iii) held. With $n=1,2$ replaced by $n \geq 1$, we would call $f$ a pgf statistic. Our previous definitions are equivalent to these when $S$ consists of paths in $G$. It is worth noting that being an icv statistic is the analogue in this discrete context of the usual notion of being increasing and concave, and being a pgf statistic is the analogue of having completely monotone derivative (see (2)).

With these stochastic orders and classes of statistics defined, we can finally state our main result:

Theorem 3. Assume that the frog paths $S_{.}(v, i)$ and counts $\eta(v)$ and $\eta^{\prime}(v)$ are mutually independent for all $v$ and $i$, and that the paths $S .(v, i)$ at a particular vertex $v$ are identically distributed for all $i$.

(a) If $f$ is a continuous icv statistic and $\eta(v) \preceq_{i c v} \eta^{\prime}(v)$ for all $v$, then $f(\eta, S) \preceq_{i c v}$ $f\left(\eta^{\prime}, S\right)$.

(b) If $f$ is a continuous pgf statistic and $\eta(v) \preceq_{p g f} \eta^{\prime}(v)$ for all $v$, then $f(\eta, S) \preceq_{p g f}$ $f\left(\eta^{\prime}, S\right)$.

The intuition behind the proof is that the extra frogs woken by the addition of two frogs at some vertex is the union of the frogs woken by the addition of each frog separately. This subadditivity property meshes neatly with concavity - for instance, the expected number of visits to the root will increase concavely as frogs are added at a vertex - and somehow this makes the frog model interact well with stochastic orders defined in terms of concave functions.

Applications. As we mentioned, our main statistic of interest fits the criteria of Theorem 3 .

Proposition 4. The count $r(\eta, S)$ of visits to $\varnothing$ in the frog model $(\eta, S)$ is a continuous icv and pgf statistic of the frog model.

This allows us to transfer many recurrence and transience results to different initial conditions. In the increasing concave order, the constant $k$ dominates all mean $k$ random variables. Theorem [3] and Proposition 4 therefore imply the following:

Corollary 5. Consider the frog model on a graph with mutually independent frog paths and i.i.d. frogs per site with common mean $\mu$. If this is almost surely recurrent, then for any integer $k \geq \mu$, the same frog model with $k$ frogs per site is almost surely recurrent.

This solves our problem of showing that the frog model on a $d$-ary tree with deterministically $k$ frogs per site is recurrent for large enough $k$. In more detail, HJJ16a, Theorem 1] establishes that on the $d$-ary tree with i.i.d.- $\operatorname{Poi}(\mu)$ frogs per site, there is a critical value 
$\mu_{c}(d)$ such that the frog model is recurrent a.s. if $\mu>\mu_{c}(d)$ and transient a.s. if $\mu<\mu_{c}(d)$. Corollary 5 , together with the estimates on $\mu_{c}(d)$ from [JJ16], give us the following result:

Corollary 6. For any $d \geq 2$, the frog model on $\mathbb{T}_{d}$ with $k$ frogs per site is almost surely recurrent for large enough $k$. For large enough $d$, the model is almost surely transient if $k<.24 d$ and almost surely recurrent if $k>2.28 d$.

Another application of Theorem 3 concerns the transience regime of the $d$-ary tree. In HJJ16b, Theorem 1] we show that on $\mathbb{T}_{d}$ with one frog per site and simple random walk paths, the frog model is transient for $d \geq 5$. An immediate corollary of Theorem 3 is transience for all other mean 1 configurations.

Corollary 7. For $d \geq 5$, the frog model on $\mathbb{T}_{d}$ with $\eta(v)$ frogs at each site, where $\mathbf{E} \eta(v) \leq 1$ for all $v \in \mathbb{T}_{d}$, is almost surely transient.

Our next application is to the frog model on $\mathbb{Z}^{d}$. As mentioned earlier, Pop01, Theorem 1.1] establishes the existence of a critical parameter $0<\alpha_{c}(d)<\infty$ for the frog model with simple random walk paths on $\mathbb{Z}^{d}$ and initial configuration given by $\eta(x) \sim \operatorname{Bernoulli}\left(p_{x}\right)$ such that

(i) if $p_{x} \leq \alpha /\|x\|^{2}$ for $\alpha<\alpha_{c}(d)$ and all sufficiently large $x$, then the model is transient with positive probability;

(ii) if $p_{x} \geq \alpha /\|x\|^{2}$ for $\alpha>\alpha_{c}(d)$ and all sufficiently large $x$, then the model is transient with probability zero.

Theorem 3 allows us to extend part (i) of this result to non-Bernoulli distributions of sleeping frogs. Other results like Pop01, Theorem 1.3] can be similarly extended.

Corollary 8. For all $\alpha<\alpha_{c}(d)$ and any $\left(\eta(x), x \in \mathbb{Z}^{d} \backslash\{0\}\right)$ satisfying $\mathbf{E} \eta(x) \leq \alpha /\|x\|^{2}$ for sufficiently large $x$, the frog model on $\mathbb{Z}^{d}$ with simple random walk paths and initial configuration $\eta$ is transient with positive probability.

A fundamental result for the frog model on $\mathbb{Z}^{d}$ is that it has a limiting shape, in the following sense. Let $\xi_{n}$ be the set consisting of all lattice squares $x+(-1 / 2,1 / 2]^{d}$ such that $x \in \mathbb{Z}^{d}$ has been visited by time $n$ in a frog model with i.i.d.- $\pi$ frogs per vertex. Theorem 1.1 from AMPR01] establishes that for any dimension $d \geq 1$, there is a convex set $\mathbf{A} \subseteq \mathbb{R}^{d}$ depending on the distribution $\pi$ such that for any $0<\epsilon<1$,

$$
\mathbf{P}\left[(1-\epsilon) \mathbf{A} \subseteq \frac{\xi_{n}}{n} \subseteq(1+\epsilon) \mathbf{A} \text { for all sufficiently large } n\right]=1 .
$$

Similar results were proven earlier for the discrete- and continuous-time model with one frog per site in AMP02a and RS04, respectively. We show that the limiting shape, A, respects the icv and pgf orders. This mirrors the inequalities for the time constant for first passage percolation that are proven in vdBK93.

Corollary 9. Let $\mathbf{A}$ and $\mathbf{A}^{\prime}$ be the limiting shapes in the above sense for a frog model on $\mathbb{Z}^{d}$ with i.i.d.- $\pi$ and i.i.d.- $\pi^{\prime}$ particles at each site, respectively. If $\pi \preceq_{\text {pgf }} \pi^{\prime}$, then $\mathbf{A} \subseteq \mathbf{A}^{\prime}$.

We also find applications to the frog model with death, explored in AMP02b, FMS04, LMP05, where frogs have an independent chance $1-p$ of dying at each step. This is a frog model according to our general definition, taking the frog paths to be stopped random walks. In this setting, the statistic of interest has been the total number of sites visited, which undergoes a phase transition on the regular tree from being finite a.s. to being infinite with positive probability as $p$ grows. The model is said to die out in the first case and to 
survive in the second. The number of sites visited is an icv and pgf statistic, as we show in Proposition 21, and we therefore obtain the following result.

Corollary 10. Let $\eta^{\prime}(v) \succeq_{\text {pgf }} \eta(v)$ be independent random variables indexed by the vertices $v$ of an arbitrary graph $G$. If the frog model with death on $G$ survives with $\eta(v)$ frogs at each $v$, then it survives with $\eta^{\prime}(v)$ frogs at each $v$.

All of the applications so far follow work with either of parts (a) and (b) of Theorem 3 , monotonicity in the icv and pgf orders, respectively. As (b) is the more difficult to prove, one might wonder why we bother with it. Our interest stems from the role of the pgf order in HJJ16b in proving recurrence for the frog model on the binary tree with one frog per site. Our argument there works by showing that the number of visits to the root is stochastically larger than any Poisson distribution in the pgf order. This hinges on HJJ16b, Lemma 10], which shows that a certain operator is monotone with respect to the pgf order. The proof there is an unsatisfying calculation that cannot easily be extended to a general $d$-ary tree. But as we explain in Remark 22 this lemma and its analogues for $d \geq 3$ are now immediate corollaries of Theorem [3(b). We hope this will be helpful in other problems such as establishing recurrence for the frog model on a 3-ary tree.

Questions. We will give a few open problems on the theme of comparison theorems. A wider range of problems on the frog model are listed in HJJ16b, HJJ16a.

We are interested in how sensitive the recurrence of the frog model is to the distribution of the frog counts. We believe that recurrence depends not just on the mean number of frogs at each vertex, but on the entire distribution.

Open Question 11. Give an example where $r(\eta, S)=\infty$ a.s. and $r\left(\eta^{\prime}, S\right)<\infty$ a.s. with $\mathbf{E} \eta(v)=\mathbf{E} \eta^{\prime}(v)$ for all $v$.

Specifically, we would like to know that with simple random walk paths on the binary tree and i.i.d.- $\pi$ frogs per vertex with mean 1 , the frog model is transient when $\pi$ is sufficiently unconcentrated.

Another question of ours is on a stronger version of Corollary 9, In vdBK93, van den Berg and Kesten prove that in first passage percolation, strictly decreasing the passage time distribution in the icv order yields a strictly smaller time constant (and hence a strictly smaller limiting shape). Most of their work is in establishing the strictness.

Open Question 12. Let $\mathbf{A}$ and $\mathbf{A}^{\prime}$ be the limiting shapes for a frog model on $\mathbb{Z}^{d}$ with i.i.d.- $\pi$ and i.i.d.- $\pi^{\prime}$ initial sleeping frogs per site, respectively. Under what conditions does it hold that $\pi \wp_{i c v} \pi^{\prime}$ implies $\mathbf{A} \subsetneq \mathbf{A}^{\prime}$ ?

This cannot hold in full generality, because all choices of $\nu$ with sufficiently heavy tails have the same limiting shape, the $L^{1}$-ball in $\mathbb{R}^{d}$ AMPR01, Theorem 1.2]. But it might hold under the assumption that $\nu$ and $\nu^{\prime}$ have finite expectations, for example. It might also hold in full generality for the continuous-time frog model, but in this setting the shape theorem has only been proven for one per site initial conditions.

Finally, we are interested in comparing frog models when the graph rather than the initial configuration is altered. As a concrete question in this vein, we ask if the $d$-regular tree is the most transient graph in the following sense:

Open Question 13. Suppose the frog model is transient on a d-regular graph $G$ with simple random walks. Is it necessarily transient on an infinite d-regular tree with simple random walk paths and the same initial conditions? 
Acknowledgments. We are grateful to Chris Hoffman for his general assistance and to Jonathan Hermon for a discussion in 2014 that mentioned Open Question 13. We thank Martin Zerner, who pointed us to the previous uses of the icv order where $X \preceq_{\text {icv }} Y$ was referred to as $X$ being more variable than $Y$. We thank Robin Pemantle, who pointed out to us the interpretation of the pgf order in terms of thinnings mentioned on page 8 . We are also grateful to Raphaël Lachièze-Rey for helping us with the French-language abstract.

\section{BACKGROUND MATERIAL ON STOCHASTIC ORDERS}

Let $\pi_{1}$ and $\pi_{2}$ be probability measures on the extended nonegative real numbers $[0, \infty]$, and let $X \sim \pi_{1}$ and $Y \sim \pi_{2}$. The following three stochastic orders play a role in this paper:

Standard stochastic order: $\pi_{1} \preceq_{\text {st }} \pi_{2}$ if $\mathbf{E} f(X) \leq \mathbf{E} f(Y)$ for all bounded increasing functions $f:[0, \infty) \rightarrow \mathbb{R}$, with $f(\infty)$ taken as $\lim _{x \rightarrow \infty} f(x)$.

Increasing concave order: $\pi_{1} \preceq_{\mathrm{icv}} \pi_{2}$ if $\mathbf{E} f(X) \leq \mathbf{E} f(Y)$ for all bounded increasing concave functions $f:[0, \infty) \rightarrow \mathbb{R}$, with $f(\infty)$ taken as $\lim _{x \rightarrow \infty} f(x)$.

Probability generating function order: $\pi_{1} \preceq_{\text {pgf }} \pi_{2}$ if $\mathbf{E} t^{X} \geq \mathbf{E} t^{Y}$ for all $t \in(0,1)$, with $t^{\infty}$ interpreted as 0 .

We use $X \preceq_{\text {st }} Y, X \preceq_{\text {st }} \pi_{2}$, and $\pi_{1} \preceq_{\text {st }} Y$ all to mean that $\pi_{1} \preceq_{\text {st }} \pi_{2}$, and we do the same with the other two orders.

We have listed these three stochastic orders in decreasing strength. That is,

$$
\pi_{1} \preceq_{\mathrm{st}} \pi_{2} \Longrightarrow \pi_{1} \preceq_{\mathrm{icv}} \pi_{2} \Longrightarrow \pi_{1} \preceq_{\mathrm{pgf}} \pi_{2} .
$$

The first implication is obvious. For the second, the map $x \mapsto 1-t^{x}$ is a bounded increasing concave function for any $t \in(0,1)$, establishing that $\mathbf{E} t^{X} \geq \mathbf{E} t^{Y}$ for $t \in(0,1)$ if $X \preceq_{\text {icv }} Y$.

See [SS07] for a reference on stochastic dominance. We have made a few slight changes from the usual definitions found there. First, in the standard and icv orders, we have required our test functions to be bounded. This apparently weaker definition is in fact equivalent to the usual one, as seen by approximating an unbounded increasing or increasing concave function by a sequence of bounded ones. Second, we have restricted ourselves to probability measures supported on nonnegative numbers, which is just a convenience. Last, we have allowed our probability measures to take the value $\infty$ with positive probability. All of the standard results on stochastic orderings are unaffected by this change. It is worth noting that if $X \preceq_{\text {pgf }} Y$, then $\mathbf{P}[X=\infty] \leq \mathbf{P}[Y=\infty]$. To see this, note that as $t \nearrow 1$, we have $t^{x} \rightarrow \mathbf{1}\{x<\infty\}$. Thus, by the monotone convergence theorem,

$$
\mathbf{E} t^{X} \rightarrow \mathbf{P}[X<\infty] \quad \text { and } \quad \mathbf{E} t^{Y} \rightarrow \mathbf{P}[Y<\infty]
$$

as $t \nearrow 1$. Now $\mathbf{E} t^{X} \geq \mathbf{E} t^{Y}$ for $t \in(0,1)$ implies that $\mathbf{P}[X<\infty] \geq \mathbf{P}[Y<\infty]$. By (1), the conclusion also holds under the assumption $X \preceq_{\text {st }} Y$ or $X \preceq_{\text {icv }} Y$. We also mention that a similar argument with a limit as $t \searrow 0$ shows that if $X \preceq_{\text {pgf }} Y$, then $\mathbf{P}[X=0] \geq \mathbf{P}[Y=0]$.

Roughly speaking, the standard order rewards distributions for being large, while the icv order rewards them either for being large or for being concentrated. The characterizations of these two orders in terms of couplings make this more precise: $X \preceq_{\text {st }} Y$ if and only if $X$ and $Y$ can be coupled so that $X \leq Y$ a.s. [SS07, Theorem 1.A.1], and $X \preceq_{\text {icv }} Y$ if and only if $X$ and $Y$ can be coupled so that $\mathbf{E}[X \mid Y] \leq Y$ a.s. [SS07, Theorem 4.A.5]. Another useful equivalent condition for $\pi_{1} \preceq_{\mathrm{st}} \pi_{2}$ is that $\mathbf{P}[X>t] \leq \mathbf{P}[Y>t]$ for all $t$.

A function $\varphi$ is called completely monotone if it is infinitely differentiable and

$$
(-1)^{n} \varphi^{(n)}(x) \geq 0
$$


for all $n \geq 0$ and $x$ in the domain of the function. By Bernstein's characterization of the completely monotone functions as mixtures of functions of the form $e^{-u x}$, the statement $X \preceq_{\text {pgf }} Y$ holds if and only if $\mathbf{E} \varphi(X) \geq \mathbf{E} \varphi(Y)$ for all completely monotone functions $\varphi$ on $[0, \infty)$, or equivalently if $\mathbf{E} \varphi(X) \leq \mathbf{E} \varphi(Y)$ for $\varphi$ with completely monotone derivative [SS07, Theorem 5.A.3]. Unlike the other two orders, the pgf order does not have a characterization in terms of couplings as far as we know, although it does have a probabilistic interpretation in terms of thinnings. The p-thinning of a nonnegative integer-valued random variable $N$ is a random variable with the conditional distribution $\operatorname{Bin}(N, p)$ given $N$. If $X$ and $Y$ are integer-valued, then $X \preceq_{\text {pgf }} Y$ if and only if the $p$-thinning of $X$ is more likely than the $p$ thinning of $Y$ to be zero, for any $p \in[0,1]$. The advantage of the pgf order in our experience is that one can test it by explicit calculations, as we did in our proof of recurrence of the one-per-site frog model on the binary tree in HJJ16b.

We now give a pair of standard propositions, whose proofs we include in the appendix for the sake of completeness. The first proposition lets us use test functions for icv and pgf dominance defined only on the nonnegative integers rather than on all of $[0, \infty)$. For a function $f$ on the integers, we define the difference operator

$$
D f(k)=f(k+1)-f(k) .
$$

We call this $D$ rather than the more common $\Delta$ to avoid ambiguity with a related operator we will define in Section 3 . Repeated application of $D$ yields the following expression (see [Sta12, eq. (1.97)]):

$$
D^{n} f(k)=\sum_{i=0}^{n}(-1)^{n-i}\left(\begin{array}{c}
n \\
i
\end{array}\right) f(k+i)
$$

Proposition 14. Let $X$ and $Y$ take values on the extended nonnegative integers. In the following statements, we assume that $\varphi(k)$ is a bounded function on the nonnegative integers with a limit as $k \rightarrow \infty$, and we interpret $\varphi(\infty)$ as this limit.

(a) It holds that $X \preceq_{i c v} Y$ if and only if $\mathbf{E} \varphi(X) \leq \mathbf{E} \varphi(Y)$ for functions $\varphi$ as above that satisfy $D \varphi(k) \geq 0$ and $D^{2} \varphi(k) \leq 0$ for all $k \geq 0$.

(b) It holds that $X \preceq_{p g f} Y$ if and only if $\mathbf{E} \varphi(X) \leq \mathbf{E} \varphi(Y)$ for functions $\varphi$ as above that satisfy $(-1)^{n} D^{n} \varphi(k) \leq 0$ for all $n \geq 1$ and $k \geq 0$.

The next proposition shows that the maximal real- and integer-valued distributions in the icv order with a given expectation are the distributions that are as concentrated as possible.

\section{Proposition 15.}

(a) A nonnegative random variable satisfies $X \preceq_{i c v} c$ for any $c \geq \mathbf{E} X$.

(b) Suppose $X$ takes nonnegative integer values and $\mathbf{E} X \in[k, k+1]$ for an integer $k$. Let $Y$ be a random variable taking values in $\{k, k+1\}$ and satisfying $\mathbf{E} X \leq \mathbf{E} Y$. Then $X \preceq_{i c v} Y$.

\section{Proof of the Comparison theorem}

We start by giving some technical facts about icv and pgf statistics and about the operator $\Delta_{P_{\text {. }}}$. As with the related operator $D$ defined in (3), this operator can be applied repeatedly and expanded in the following way, which resembles (4). Let $P_{\bullet}^{1}, \ldots, P_{\bullet}^{n}$ be frog paths, and let $U=\left\{u_{1}, \ldots, u_{j}\right\} \subseteq[n]$, where we use the notation $[n]=\{1, \ldots, n\}$. Define

$$
\sigma_{U}(\eta, S)=\sigma_{P_{\bullet}^{u_{1}}} \cdots \sigma_{P_{\bullet}^{u_{j}}}(\eta, S)
$$


the frog model $(\eta, S)$ with the addition of frogs $P_{\bullet}^{u_{1}}, \ldots, P_{\bullet}^{u_{j}}$. If $U$ is empty, take $\sigma_{U}(\eta, S)=$ $(\eta, S)$. Using this notation,

$$
\Delta_{P_{\bullet}^{1}} \cdots \Delta_{P_{\bullet}^{n}} f(\eta, S)=\sum_{U \subseteq[n]}(-1)^{n-|U|} f\left(\sigma_{U}(\eta, S)\right) .
$$

This can be proven by the same argument used in [Sta12, eq. (1.97)].

Our next proposition states that icv statistics are closed under composition with increasing concave functions, and that pgf statistics are closed under composition with functions with completely monotone derivatives. Though we could not find these statements in existing literature, they are essentially equivalent to the well known fact that increasing concave functions and functions with completely monotone derivatives both form closed families under composition. We give a full proof in the appendix.

Proposition 16. Let $\varphi:[0, \infty) \rightarrow[0, \infty)$ be bounded, and interpret $\varphi(\infty)$ as $\lim _{x \rightarrow \infty} \varphi(x)$.

(a) Suppose that $\varphi$ is increasing and concave. If $f$ is an icv statistic, then $\varphi \circ f$ is also an icv statistic.

(b) Suppose that $\varphi$ has completely monotone derivative; that is, it satisfies

$$
(-1)^{n} \varphi^{(n)}(x) \leq 0
$$

for all $n \geq 1$. If $f$ is a pgf statistic, then $\varphi \circ f$ is also a pgf statistic.

In the next lemma, we show that icv and pgf statistics are monotone in the distribution of frogs at a single vertex.

Lemma 17. Make the assumptions of Theorem 3 on the distribution of frog paths $S .(v, i)$ and counts $\eta(v)$ and $\eta^{\prime}(v)$. Also assume that $\eta$ and $\eta^{\prime}$ have identical distributions at all but one vertex $v_{0}$.

(a) If $f$ is an icv statistic and $\eta\left(v_{0}\right) \preceq_{i c v} \eta^{\prime}\left(v_{0}\right)$, then $f(\eta, S) \preceq_{i c v} f\left(\eta^{\prime}, S\right)$.

(b) If $f$ is a pgf statistic and $\eta\left(v_{0}\right) \preceq_{p g f} \eta^{\prime}\left(v_{0}\right)$, then $f(\eta, S) \preceq_{p g f} f\left(\eta^{\prime}, S\right)$.

Proof. Define $\eta_{k}$ to be the same as $\eta$ except that $\eta_{k}\left(v_{0}\right)=k$. Let $W(k)=f\left(\eta_{k}, S\right)$. By our assumptions, $\eta\left(v_{0}\right)$ and $\eta^{\prime}\left(v_{0}\right)$ are independent of $W(k)$, and hence

$$
W\left(\eta\left(v_{0}\right)\right) \sim f(\eta, S) \quad \text { and } \quad W\left(\eta^{\prime}\left(v_{0}\right)\right) \sim f\left(\eta^{\prime}, S\right) .
$$

We start with the proof of $(\mathrm{a})$. Let $\varphi:[0, \infty) \rightarrow[0, \infty)$ be an arbitrary bounded increasing concave function, and let $h(k)=\mathbf{E} \varphi(W(k))$ for $k \in\{0,1, \ldots\}$. As

$$
\mathbf{E} h\left(\eta\left(v_{0}\right)\right)=\mathbf{E} \varphi(f(\eta, S)) \quad \text { and } \quad \mathbf{E} h\left(\eta^{\prime}\left(v_{0}\right)\right)=\mathbf{E} \varphi\left(f\left(\eta^{\prime}, S\right)\right)
$$

by (17), our goal is to show that $\mathbf{E} h\left(\eta\left(v_{0}\right)\right) \leq \mathbf{E} h\left(\eta^{\prime}\left(v_{0}\right)\right)$. If we can show that $D h(k) \geq 0$ and $D^{2} h(k) \leq 0$, then this follows immediately from Proposition 14)(a) and the assumption that $\eta\left(v_{0}\right) \preceq_{\text {icv }} \eta^{\prime}\left(v_{0}\right)$.

By definition of $h$ and $W$,

$$
D h(k)=\mathbf{E}\left[\varphi\left(f\left(\eta_{k+1}, S\right)\right)-\varphi\left(f\left(\eta_{k}, S\right)\right)\right] .
$$

As $f\left(\eta_{k+1}, S\right) \geq f\left(\eta_{k}, S\right)$ and $\varphi$ is increasing, one can see directly that $D h(k) \geq 0$, but it is more instructive to derive this as a consequence of Proposition 16, Let $P$. be an independent copy of $S \cdot\left(v_{0}, 1\right)$. By our assumption that $\left(S .\left(v_{0}, i\right)\right)_{i \geq 1}$ are i.i.d. and independent of the other frog paths, the frog model $\left(\eta_{k+1}, S\right)$ is distributed the same as $\sigma_{P_{\mathbf{0}}}\left(\eta_{k}, S\right)$. Hence

$$
\begin{aligned}
D h(k) & =\mathbf{E}\left[\varphi\left(f\left(\sigma_{P_{\bullet}}\left(\eta_{k}, S\right)\right)-\varphi\left(f\left(\eta_{k}, S\right)\right)\right]\right. \\
& =\mathbf{E}\left[\Delta_{P_{\bullet}}(\varphi \circ f)\left(\eta_{k}, S\right)\right],
\end{aligned}
$$


which is nonnegative since $\varphi \circ f$ is an icv statistic by Proposition 1f(a) Similarly, if $P^{1}$ and $P_{\bullet}^{2}$ are independent copies of $S_{\bullet}\left(v_{0}, i\right)$,

(9)

$$
\begin{aligned}
D^{2} h(k) & =\mathbf{E}\left[\varphi\left(f\left(\eta_{k+2}, S\right)\right)-2 \varphi\left(f\left(\eta_{k+1}, S\right)\right)+\varphi\left(f\left(\eta_{k}, S\right)\right)\right] \\
& =\mathbf{E}\left[\varphi\left(f\left(\sigma_{P_{\bullet}^{1}} \sigma_{P^{2}}\left(\eta_{k}, S\right)\right)\right)-\varphi\left(f\left(\sigma_{P_{\bullet}^{1}}\left(\eta_{k}, S\right)\right)\right)-\varphi\left(f\left(\sigma_{P_{\bullet}^{2}}\left(\eta_{k}, S\right)\right)\right)+\varphi\left(f\left(\eta_{k}, S\right)\right)\right] \\
& =\mathbf{E}\left[\Delta_{P_{\bullet}^{1}} \Delta_{P_{\bullet}^{2}}(\varphi \circ f)\left(\eta_{k}, S\right)\right] \leq 0 .
\end{aligned}
$$

This concludes the proof of part (a).

The proof of (b) is essentially the same. We take $\varphi(x)=1-t^{x}$ for arbitrary $t \in(0,1)$ and define $h(k)=\mathbf{E} \varphi(W(k))$ as before. This time, we need to show that $(-1)^{n} D^{n} h(k) \leq 0$ for all $n \geq 1$. By Proposition 14](b) and the assumption that $\eta\left(v_{0}\right) \preceq_{\text {pgf }} \eta^{\prime}\left(v_{0}\right)$, it follows from this that $\mathbf{E} h\left(\eta\left(v_{0}\right)\right) \leq \mathbf{E} h\left(\eta^{\prime}\left(v_{0}\right)\right)$, and hence that $\mathbf{E} \varphi(f(\eta, S)) \leq \mathbf{E} \varphi\left(f\left(\eta^{\prime}, S\right)\right)$.

Let $P_{\bullet}^{1}, \ldots, P_{\bullet}^{n}$ be independent copies of $S \cdot\left(v_{0}, i\right)$. Using the notation of (5), for any $U \subseteq[n]$ with $|U|=i$, the frog model $\left(\eta_{k+i}, S\right)$ is distributed identically to $\sigma_{U}\left(\eta_{k}, S\right)$. We now generalize (8) and (9) by applying (44) and (6) to get

$$
\begin{aligned}
D^{n} h(k) & =\mathbf{E}\left[\sum_{i=0}^{n}(-1)^{n-i}\left(\begin{array}{c}
n \\
i
\end{array}\right) \varphi\left(f\left(\eta_{k+i}, S\right)\right)\right] \\
& =\mathbf{E}\left[\sum_{i=0}^{n}(-1)^{n-i} \sum_{\substack{U \subseteq[n] \\
|U|=i}} \varphi\left(f\left(\sigma_{U}\left(\eta_{k}, S\right)\right)\right)\right] \\
& =\mathbf{E}\left[\Delta_{P_{\bullet}^{1}} \cdots \Delta_{P_{\bullet}^{n}}(\varphi \circ f)\right] .
\end{aligned}
$$

As $\varphi \circ f$ is a pgf statistic by Proposition 16(b), this shows that $(-1)^{n} D^{n} h(k) \leq 0$, completing the proof.

Proof of Theorem 3. The basic idea is that Lemma 17proves the result when $\eta$ and $\eta^{\prime}$ have the same distribution at all but finitely many vertices, with the general case following from a limit argument relying on the continuity assumption. Recall from Definition 1 that we call a frog model statistic continuous if the upward convergence of frog counts implies the upward convergence of the statistic.

Let $G_{1} \subseteq G_{2} \subseteq \cdots$ be finite sets of vertices whose union is $G$. We use $\left.\eta\right|_{G_{k}}$ and $\left.\eta^{\prime}\right|_{G_{k}}$ to denote restrictions to $G_{k}$. That is, $\left.\eta\right|_{G_{k}}(v)=\eta(v) \mathbf{1}\left\{v \in G_{k}\right\}$. Since $\left.\eta\right|_{G_{k}}$ and $\left.\eta^{\prime}\right|_{G_{k}}$ differ at only finitely many vertices, the repeated application of Lemma 17 proves that in case (a).

$$
f\left(\left.\eta\right|_{G_{k}}, S\right) \preceq_{\mathrm{icv}} f\left(\left.\eta^{\prime}\right|_{G_{k}}, S\right),
$$

and in case (b),

$$
f\left(\left.\eta\right|_{G_{k}}, S\right) \preceq_{\text {pgf }} f\left(\left.\eta^{\prime}\right|_{G_{k}}, S\right) .
$$

Now, we let $\varphi$ be a test function and try to show that

$$
\mathbf{E} \varphi(f(\eta, S)) \leq \mathbf{E} \varphi\left(f\left(\eta^{\prime}, S\right)\right) .
$$

For case (a), let $\varphi:[0, \infty) \rightarrow[0, \infty)$ be a bounded increasing concave function. It suffices to show (12) for such functions $f$, as any arbitrary bounded increasing concave function can be shifted to take nonnegative values. In case (b), let $\varphi(x)=1-t^{x}$ for some $t \in(0,1)$. Interpreting $\varphi(\infty)$ as $\lim _{x \rightarrow \infty} \varphi(x)$ as usual, it holds by the continuity assumption that

$$
\varphi\left(f\left(\left.\eta\right|_{G_{k}}, S\right)\right) \nearrow \varphi(f(\eta, S)) \text { a.s. } \quad \text { and } \quad \varphi\left(f\left(\left.\eta^{\prime}\right|_{G_{k}}, S\right)\right) \nearrow \varphi\left(f\left(\eta^{\prime}, S\right)\right) \text { a.s. }
$$


as $k \rightarrow \infty$. By the monotone convergence theorem,

$$
\mathbf{E} \varphi\left(f\left(\left.\eta\right|_{G_{k}}, S\right)\right) \rightarrow \mathbf{E} \varphi(f(\eta, S)) \quad \text { and } \quad \mathbf{E} \varphi\left(f\left(\left.\eta^{\prime}\right|_{G_{k}}, S\right)\right) \rightarrow \mathbf{E} \varphi\left(f\left(\eta^{\prime}, S\right)\right)
$$

as $k \rightarrow \infty$. By (10) or (11), we have $\mathbf{E} \varphi\left(f\left(\left.\eta\right|_{G_{k}}, S\right)\right) \leq \mathbf{E} \varphi\left(f\left(\left.\eta^{\prime}\right|_{G_{k}}, S\right)\right)$, and this proves (12).

\section{Applications of the Comparison theorem}

To apply Theorem 3, we first need to find some icv and pgf statistics. The following two lemmas highlight particular circumstances where we can draw conclusions about the difference operators applied to a statistic. For any frog model $(\eta, S)$, we define $\kappa_{v}(\eta, S)$ as the frog model resulting from deleting all frogs that start at $v$ from $(\eta, S)$. Formally, $\kappa_{v}(\eta, S)=\left(\eta^{\prime}, S\right)$, where $\eta^{\prime}$ is identical to $\eta$ except that $\eta^{\prime}(v)=0$.

Lemma 18. Let $f$ be a frog model statistic taking values only in $\{0,1\}$. Suppose that for some vertex $v$ and all $(\eta, S)$,

$$
f(\eta, S)=\max \left\{f\left(\kappa_{v}(\eta, S)\right), f\left(\sigma_{S_{\bullet}}\left(\kappa_{v}(\eta, S)\right)\right), \ldots, f\left(\sigma_{S_{\bullet}^{\eta(v)}}\left(\kappa_{v}(\eta, S)\right)\right)\right\}
$$

Then for any paths $P_{\bullet}^{1}, \ldots, P_{\bullet}^{n}$ originating at $v$ and all $(\eta, S)$,

$$
(-1)^{n} \Delta_{P_{\bullet}^{1}} \cdots \Delta_{P_{\bullet}^{n}} f(\eta, S) \leq 0 .
$$

Proof. Fix any $(\eta, S)$ and paths $P_{\bullet}^{1}, \ldots, P_{\bullet}^{n}$ starting at $v$. Since $f$ is increasing as additional frogs are added by (13), if $f(\eta, S)=1$, then $f\left(\sigma_{U}(\eta, S)\right)=1$ for any $U \subseteq[n]$, using the notation given in (5). Hence

$$
\Delta_{P \cdot} \cdots \Delta_{P_{\bullet}^{n}} f(\eta, S)=0
$$

and so the lemma holds in this case. If $f(\eta, S)=0$, define $b_{i}=f\left(\sigma_{P_{*}^{i}}(\eta, S)\right)$, the statistic after adding $P_{\bullet}^{i}$ to $(\eta, S)$. By (13), $f\left(\sigma_{U}(\eta, S)\right)=1$ if and only if $b_{i}=1$ for some $i \in U$. Thus

$$
f\left(\sigma_{U}(\eta, S)\right)=\max _{i \in U} b_{i}=1-\prod_{i \in U}\left(1-b_{i}\right)
$$

and (6) gives

$$
\begin{aligned}
\Delta_{P \cdot} \cdots \Delta_{P_{\bullet}^{n}} f(\eta, S) & =\sum_{U \subseteq[n]}(-1)^{n-|U|}\left(1-\prod_{i \in U}\left(1-b_{i}\right)\right) \\
& =\sum_{U \subseteq[n]}(-1)^{n-|U|}-\sum_{U \subseteq[n]}(-1)^{n-|U|} \prod_{i \in U}\left(1-b_{i}\right) .
\end{aligned}
$$

The first sum is the expansion of $(1-1)^{n}$ and hence is zero. For the second sum,

$$
\begin{aligned}
\sum_{U \subseteq[n]}(-1)^{n-|U|} \prod_{i \in U}\left(1-b_{i}\right) & =\prod_{i=1}^{n}\left(\left(1-b_{i}\right)-1\right) \\
& =\prod_{i=1}^{n}\left(-b_{i}\right)=(-1)^{n} \mathbf{1}\left\{b_{1}=\cdots=b_{n}=1\right\} .
\end{aligned}
$$

Thus

$$
\Delta_{P_{\bullet}^{1}} \cdots \Delta_{P_{\bullet}^{n}} f(\eta, S)=(-1)^{n+1} \mathbf{1}\left\{b_{1}=\cdots=b_{n}=1\right\},
$$

yielding $(-1)^{n} \Delta_{P_{\bullet}^{1}} \cdots \Delta_{P_{\bullet}^{n}} f(\eta, S) \leq 0$. 
Lemma 19. Let $f$ be a frog model statistic taking values in $[0, \infty]$. Suppose that for some vertex $v$ and all $(\eta, S)$,

$$
f(\eta, S)=\sum_{i=1}^{\eta(v)} f\left(\sigma_{S_{\bullet}(v, i)}\left(\kappa_{v}(\eta, S)\right)\right) .
$$

Then conditions (i), (ii), and (iii) of being a pgf statistic hold for all $(\eta, S)$ and paths $P_{\bullet}^{1}, \ldots, P_{\bullet}^{n}$ originating at $v$.

Proof. Fix $(\eta, S)$ and paths $P_{\bullet}^{1}, \ldots, P_{\bullet}^{m}$ originating at $v$. Let $b_{i}=f\left(\sigma_{P_{*}^{i}}\left(\kappa_{v}(\eta, S)\right)\right)$, and let $b=f(\eta, S)$. As in the previous lemma, we use the notation $\sigma_{U}$ given in (5). From (14), for any $U \subseteq[m]$,

$$
f\left(\sigma_{U}(\eta, S)\right)=b+\sum_{i \in U} b_{i} .
$$

First, consider the case that $b, b_{1}, \ldots, b_{m}<\infty$. Let

$$
D_{a} h(x)=h(x+a)-h(x)
$$

generalizing the difference operator $D$ given in (3). The operator $D_{a}$ satisfies

$$
D_{a_{1}} \cdots D_{a_{m}} h(x)=\sum_{U \subseteq[m]}(-1)^{m-|U|} h\left(x+\sum_{i \in U} a_{i}\right),
$$

proven identically as (4) and (6). Comparing with (6), we have

$$
\Delta_{P_{\bullet}^{1}} \cdots \Delta_{P_{\bullet}^{m}} f(\eta, S)=D_{b_{1}} \cdots D_{b_{m}} \mathrm{id}(b),
$$

where $\operatorname{id}(x)=x$. For $m \geq 2$, this is equal to zero, as follows from the second and higher partial derivatives of id being zero. For $m=1$, this is nonnegative, because $b_{1} \geq 0$ by our assumption that $f$ takes nonnegative values. This shows that condition (i) holds. Conditions (ii) and (iii) follow immediately from (14).

Next, we show that icv and pgf statistics are closed under summation.

Lemma 20. Let $f_{1}, f_{2}, \ldots$ be frog model statistics taking values in $[0, \infty]$, and let $f=$ $\sum_{i=1}^{\infty} f_{i}$. If $f_{1}, f_{2}, \ldots$ are icv statistics, then $f$ is an icv statistic, and if $f_{1}, f_{2}, \ldots$ are pgf statistics, then $f$ is a pgf statistic.

Proof. Suppose that $f_{1}, f_{2}, \ldots$ are pgf statistics. We have

$$
(-1)^{m} \Delta_{P_{\bullet}^{1}} \cdots \Delta_{P_{\bullet}^{m}} f(\eta, S)=\sum_{i=1}^{\infty}(-1)^{m} \Delta_{P_{\bullet}^{1}} \cdots \Delta_{P_{\bullet}^{m}} f_{i}(\eta, S)
$$

when all quantities in the expansion of the left hand side are finite. All quantities on the right hand side are finite as well, and all are nonpositive since $f_{i}$ is a pgf statistic for all $i$, which shows that condition (i) is satisfied. For condition (ii) we note that by conditions (i) and (ii) applied to $f_{i}$,

$$
\sum_{i=1}^{\infty} f_{i}(\eta, S) \leq \sum_{i=1}^{\infty} f_{i}\left(\sigma_{P_{\bullet}^{1}}(\eta, S)\right)
$$


even when the left hand side is infinite. And for condition (iii), by conditions (i) and (iii) applied to $f_{i}$

$$
\sum_{i=1}^{\infty} f_{i}\left(\sigma_{P_{\bullet}^{1}} \sigma_{P_{\bullet}^{2}}(\eta, S)\right) \leq \sum_{i=1}^{\infty} f_{i}\left(\sigma_{P_{\bullet}^{1}}(\eta, S)\right)+\sum_{i=1}^{\infty} f_{i}\left(\sigma_{P_{\bullet}^{2}}(\eta, S)\right),
$$

even when the left hand side is infinite. The proof is identical for the icv case.

Now, we apply Lemmas 18 and 19 to prove that various frog model statistics are pgf (and hence also icv). We start with some counts of visited sites.

Proposition 21. For $t \in \mathbb{N} \cup\{\infty\}$ and any nonroot vertex $u$, let $a_{t, u}(\eta, S)$ be an indicator on site $u$ being visited in the frog model $(\eta, S)$ by time $t$. Let $a_{t}(\eta, S)$ be the total number of nonroot sites visited by time $t$. Then both $a_{t, u}$ and $a_{t}$ are continuous icv and pgf statistics.

Proof. First, we show that $a_{t, u}$ is a pgf statistic. Let $v \neq u$ be a nonroot vertex. We claim that (13) is satisfied for $a_{t, u}$ and vertex $v$. To prove this, we need to show that $a_{t, u}(\eta, S)=1$ if and only if $a_{t, u}\left(\kappa_{v}(\eta, S)\right)=1$ or $a_{t, u}\left(\sigma_{S \cdot(v, i)}\left(\kappa_{v}(\eta, S)\right)\right)=1$ for some $1 \leq i \leq \eta(v)$. First, suppose $a_{t, u}(\eta, S)=1$. This means that there exists a sequence of frogs starting with the initial frog and ending with a frog that visits $u$ such that each frog activates the next one in the sequence and the combined path length is at most $t$. We can assume that all frogs in this sequence originate at different vertices, since otherwise we could cut out portions of the sequence to make this true. If the sequence includes the $i$ th frog at $v$, then $a_{t, u}\left(\sigma_{S \cdot(v, i)}\left(\kappa_{v}(\eta, S)\right)\right)=1$. If the sequence does not include any of the frogs originating at $v$, then $a_{t, u}\left(\kappa_{v}(\eta, S)\right)=1$. The converse is obvious, since if $u$ is visited in time $t$ by $\sigma_{S .(v, i)}\left(\kappa_{v}(\eta, S)\right)$ or $\kappa_{v}(\eta, S)$, then it is also visited by $(\eta, S)$.

Thus, Lemma 18 applies and shows that

$$
(-1)^{n} \Delta_{P_{\bullet}^{1}} \cdots \Delta_{P_{\bullet}^{n}} a_{t, u}(\eta, S) \leq 0
$$

for any $(\eta, S)$, nonroot vertices $u \neq v$, and paths $P_{\bullet}^{1}, \ldots, P_{\bullet}^{n}$ originating at $v$. In the case $u=v$,

$$
(-1)^{n} \Delta_{P_{\bullet}^{1}} \cdots \Delta_{P_{\bullet}^{n}} a_{t, u}(\eta, S)=0,
$$

as the addition of extra frogs at $u$ does not affect whether $u$ is visited. Thus, $a_{t, u}$ is a pgf statistic for any nonroot vertex $u$. It follows by Lemma 20 that $a_{t}$ is a pgf statistic, since we can express it as

$$
a_{t}(\eta, S)=\sum_{v \neq \varnothing} a_{t, v}(\eta, S)
$$

It remains to prove that $a_{t, u}$ and $a_{t}$ are continuous. This holds because any frog woken in $(\eta, S)$ relies only on a finite sequence of frogs to wake it. More formally, suppose that the components of $\eta_{k}$ converge upwards to $\eta$ as $k \rightarrow \infty$. If $a_{t, u}(\eta, S)=1$, then for large enough $k$ we have $a_{t, v}\left(\eta_{k}, S\right)=1$, because the sequence of frogs visiting $u$ in time $t$ is finite. Thus $a_{t, u}\left(\eta_{k}, S\right) \nearrow a_{t, u}(\eta, S)$ as $k \rightarrow \infty$, meaning that $a_{t, u}$ is continuous. By (15) and monotone convergence, $a_{t}\left(\eta_{k}, S\right) \nearrow a_{t}(\eta, S)$, and $a_{t}$ is continuous as well.

Proof of Proposition 4, This is a slightly more complicated version of the previous proof. For any nonroot vertex $u$, let $r_{u}(\eta, S)$ be the number of visits to the root in the frog model $(\eta, S)$ by frogs originating at vertex $u$. Fix some vertex $v \neq u$ and frog paths $P_{\bullet}^{1}, \ldots, P_{\bullet}^{m}$ originating at $v$, and fix $(\eta, S)$. Let $N$ be the total number of visits to the root by paths 
S. $(u, 1), \ldots, S_{\mathbf{*}}(u, \eta(u))$. Since adding extra frogs at vertex $v$ affects only whether $u$ is activated, not the number of frogs returning from it if activated,

$$
r_{u}\left(\sigma_{U}(\eta, S)\right)=N a_{\infty, u}\left(\sigma_{U}(\eta, S)\right)
$$

for any $U \subseteq[m]$. It then follows from Proposition 21 that $(-1)^{m} \Delta_{P_{*}^{1}} \cdots \Delta_{P_{*}^{m}} r_{u}(\eta, S) \leq 0$ when all terms in this expansion are finite (that is, when $N<\infty$ ). From the $N=\infty$ case of (16), it also follows that conditions (ii) and (iii) hold for all $(\eta, S)$ and all paths $P_{\bullet}^{1}, \ldots P_{\bullet}^{m}$ originating at $v$.

Next, we consider the case $v=u$. If $u$ is visited by $(\eta, S)$, then the number of visits to the root originating at $u$ is the number of visits to the root by paths $S \cdot(u, 1), \ldots, S \cdot(u, \eta(u))$. Since modifying the frogs at $u$ does not change whether $u$ is visited, this implies that if $a_{\infty, u}(\eta, S)=1$,

$$
r_{u}(\eta, S)=\sum_{i=1}^{\eta(u)} r_{u}\left(\sigma_{S_{\bullet}(u, i)}\left(\kappa_{v}(\eta, S)\right)\right) .
$$

This equation also holds if $a_{\infty, u}(\eta, S)=0$, since then both sides are zero. Hence the conditions of Lemma 19 are satisfied, and conditions (i), (ii), and (iii) are satisfied for all $(\eta, S)$ and all paths originating at $v$.

Thus, we have shown that $r_{u}$ is a pgf statistic. By writing $r$ as a sum of $r_{u}$ over $u \in G$, we see that $r$ is also a pgf statistic by Lemma 20, and by the same argument as in the proof of Proposition 21, this statistic is continuous.

Having established our comparison theorem and that several frog model statistics are icv and pgf statistics, we can now establish the relevant corollaries from Section 1.

Proofs of Corollaries 5 and 7 . We apply Theorem 3 , Proposition 4, and Proposition 15](a). along with the observation made in Section 2 that $\mathbf{P}[X=\infty] \leq \mathbf{P}[Y=\infty]$ if $X \preceq_{\text {icv }} Y$.

Proof of Corollary [6. The transience part of this result is a consequence of [HJJ16a, Proposition 15]. The recurrence part follows from Corollary [5, [HJJ16a, Theorem 1], and [JJ16, Theorem 1].

Proof of Corollary 8 . This is proven the same way as Corollaries 5 and 7 , except that Proposition 15.(b) is used instead of Proposition 15.(a).

Proof of Corollary 9, For $v \in \mathbb{Z}^{d}$, let $T(v)$ and $T^{\prime}(v)$ be the time that the vertex $v$ is activated for the frog models with i.i.d.- $\pi$ and i.i.d.- $\pi^{\prime}$ frogs per site, respectively. Let $a_{t, v}=\mathbf{1}\{T(v) \leq t\}$ be an indicator that $v$ has been activated by time $t$, and similarly for $a_{t, v}^{\prime}$. By Proposition 21, $a_{t, v}$ and $a_{t, v}^{\prime}$ are continuous pgf statistics. Moreover, we can express $T(v)$ and $T^{\prime}(v)$ in terms of these statistics:

$$
T(v)=\sum_{t=0}^{\infty}\left(1-a_{t, v}\right), \quad T^{\prime}(v)=\sum_{t=0}^{\infty}\left(1-a_{t, v}^{\prime}\right) .
$$

By Theorem 3, we have $a_{t, v} \preceq_{\text {pgf }} a_{t, v}^{\prime}$, and hence $\mathbf{E} a_{t, v} \leq \mathbf{E} a_{t, v}^{\prime}$. Apply this, along with Fubini's theorem, to the expressions for $T(v)$ and $T^{\prime}(v)$ to obtain

$$
\mathbf{E} T^{\prime}(v) \leq \mathbf{E} T(v) .
$$

In the proof of AMPR01, Theorem 1.1], the limiting shapes are determined by functions $\mu$ and $\mu^{\prime}$ with domain $\mathbb{R}^{d}$ defined via Kingman's subadditive ergodic theorem by

$$
\mu(v)=\lim _{n \rightarrow \infty} \frac{T(n v)}{n}=\inf _{n \geq 1} \frac{\mathbf{E} T(n v)}{n},
$$




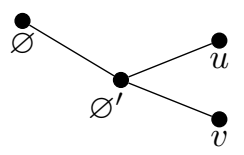

Figure 1. A graph used to define the operator $\mathcal{A}$ in HJJ16b.

where $v \in \mathbb{Z}^{d}$, with $\mu^{\prime}$ defined analogously. After interpolating to all of $\mathbb{R}^{d}$ (see Step 5 in the proof of [AMPR01, Theorem 1.1]), the limiting shape is given by $\mathbf{A}=\left\{x \in \mathbb{R}^{d}: \mu(x) \leq 1\right\}$. The set $\mathbf{A}^{\prime}$ is obtained in the same fashion. To deduce that $\mathbf{A} \subseteq \mathbf{A}^{\prime}$, it then suffices to show that $\mu^{\prime}(v) \leq \mu(v)$ for $v \in \mathbb{Z}^{d}$. This follows from (17) applied to the expected value formulation of $\mu$ in (18).

Proof of Corollary 10. This also has the same proof as Corollaries [5, 6, and 7, except that Proposition 21 replaces Proposition 4 .

Remark 22. As we mentioned in the introduction, one of the motivations for part (b) of Theorem 3 is that Lemma 10 from [HJJ16b] and similar results are direct corollaries of it. Besides providing a satisfying explanation of why Lemma 10 holds, this is potentially useful in deriving other recurrence results.

Here, we describe Lemma 10 in more detail and explain why it follows from Theorem 3 (b) The lemma is a monotonicity result for an operator $\mathcal{A}$ acting on probability distributions on the nonnegative integers. (In HJJ16b, the operator is described as acting on probability generating functions, but this comes to the same thing.) The operator can be defined as follows. Let $\pi$ be a probability distribution on the nonnegative integers. Consider a binary tree truncated to four vertices as in Figure 1. Place one frog on $\varnothing$ and one frog on $\varnothing^{\prime}$, and independently sample from $\pi$ to decide the number of frogs on $u$ and $v$. The frog paths are random nonbacktracking walks stopped when a frog reaches a leaf. Now, run the frog model starting with the frog at $\varnothing$ active until all frogs are stopped. Define $\mathcal{A} \pi$ to be the distribution of frogs terminating at $\varnothing$. In [HJJ16b, the operator was defined in a different way, but it turns out to be equivalent. See also [HJJ16a, Section 2.2] and [JJ16, Section 3.1.2] for similar constructions.

The result of Lemma 10 is that $\pi \preceq_{\text {pgf }} \pi^{\prime}$ imples that $\mathcal{A} \pi \preceq_{\text {pgf }} \mathcal{A} \pi^{\prime}$. As $\mathcal{A} \pi$ is the distribution of visits to $\varnothing$ in the frog model on the truncated graph, it is a continuous pgf statistic by Proposition 4 Thus the lemma is a consequence of Theorem 3(b).

\section{APPENDIX}

Proof of Proposition 14. If $\varphi(x)$ is increasing and concave on $[0, \infty)$, then it is easily seen that $D \varphi(k) \geq 0$ and $D^{2} \varphi(k) \leq 0$ for all $k$. Similarly, one can easily check that if $\varphi(x)=1-p^{x}$, then $(-1)^{n} D^{n} \varphi(k) \leq 0$ for all $k$. This proves that the criteria stated in (a) and (b) imply icv and pgf dominance, respectively.

For the other direction in (a), suppose that $X \preceq_{\text {icv }} Y$. Let $\varphi$ be a test function defined on the nonnegative integers satisfying $D \varphi(k) \geq 0$ and $D^{2} \varphi(k) \leq 0$ for all $k$. This can be extended to an increasing concave function on $[0, \infty)$ by linearly interpolating between integer points, for example, and hence $\mathbf{E} \varphi(X) \leq \mathbf{E} \varphi(Y)$ by the assumption that $X \preceq_{\text {icv }} Y$. 
For (b), suppose that $X \preceq_{\text {pgf }} Y$, and let $\varphi$ be a bounded test function on the nonnegative integers satisfying $(-1)^{n} D^{n} \varphi(k) \leq 0$ for all $k$. We now appeal to a classic result of Hausdorff's stating that a sequence $f(0), f(1), \ldots$ can be represented as a moment sequence

$$
f(k)=\int_{[0,1]} u^{k} \sigma(d u)
$$

for some positive measure $\sigma$ if and only if

$$
(-1)^{n} D^{n} f(k) \geq 0
$$

for all $n \geq 0$. (See [Akh65, Theorem 2.6.4], and note that $\Delta^{n}$ defined there is equal to $(-1)^{n} D^{n}$.) Let $C$ be an upper bound on $\varphi$, and apply Hausdorff's result to $C-\varphi(k)$ to obtain the representation

$$
C-\varphi(k)=\int_{[0,1]} u^{k} \sigma(d u)
$$

for some measure $\sigma$. Defining

$$
\psi(x)=\int_{[0,1]} u^{x} \sigma(d u),
$$

for $x>0$ and extending the function continuously to $x=0$, we obtain a completely monotone function $\psi$ satisfying

$$
\begin{aligned}
& \psi(k)=C-\varphi(k), \quad k \in\{1,2, \ldots\}, \\
& \psi(0)=\int_{(0,1]} \sigma(d u) \leq C-\varphi(0) .
\end{aligned}
$$

Now, we evaluate

$$
\begin{aligned}
& \mathbf{E} \varphi(X)=C-\mathbf{E} \psi(X)-\mathbf{P}[X=0](C-\psi(0)-\varphi(0)), \\
& \mathbf{E} \varphi(Y)=C-\mathbf{E} \psi(Y)-\mathbf{P}[Y=0](C-\psi(0)-\varphi(0)) .
\end{aligned}
$$

Since $\psi$ is completely monotone and $X \preceq_{\text {pgf }} Y$, we have $\mathbf{E} \psi(X) \geq \mathbf{E} \psi(Y)$. The relation $X \preceq_{\text {pgf }} Y$ implies that $\mathbf{P}[X=0] \geq \mathbf{P}[Y=0]$, and together with $C-\psi(0)-\varphi(0) \geq 0$, this implies that $\mathbf{E} \varphi(X) \leq \mathbf{E} \varphi(Y)$.

Proof of Proposition 15. Part (a) follows immediately from Jensen's inequality. For part(b), let $\varphi$ be an arbitrary increasing concave function on $[0, \infty)$. To simplify the algebra, let $U=X-k, V=Y-k$, and $\psi(x)=\varphi(x+k)-\varphi(k)$. With these replacements, our goal is to show that $\mathbf{E} \psi(U) \leq \mathbf{E} \psi(V)$. We know that $\mathbf{E} U \in[0,1]$ and that $V$ is Bernoulli, and we know that $\psi$ is increasing and concave on $[-k, \infty)$ and satisfies $\psi(0)=0$.

Since $V$ is Bernoulli with mean at least $\mathbf{E} U$,

$$
\mathbf{E} \psi(V) \geq(\mathbf{E} U) \psi(1) .
$$

Define

$$
\begin{aligned}
& a=\mathbf{E}[U \mid U \leq 0], \\
& p=\mathbf{P}[U \leq 0], \\
& b=\mathbf{E}[U \mid U \geq 1], \\
& q=1-p=\mathbf{P}[U \geq 1] .
\end{aligned}
$$

If $p=0$ or $q=0$, then $U$ is deterministic and the result is trivial because $U$ and $V$ have the same distribution. Thus we can assume that both conditional expectations above are well defined. 
Applying Jensen's inequality,

$$
\mathbf{E} \psi(U)=p \mathbf{E}[\psi(U) \mid U \leq 0]+q \mathbf{E}[\psi(U) \mid U \geq 1] \leq p \psi(a)+q \psi(b) .
$$

As $a \leq 0$ and $b \geq 1$, the points $(a, \psi(a))$ and $(b, \psi(b))$ lie under the secant line connecting $(0,0)$ and $(1, \psi(1))$ by the concavity of $\psi$. Thus $\psi(a) \leq a \psi(1)$ and $\psi(b) \leq b \psi(1)$. Applying this to (20) and combining with (19) gives

$$
\mathbf{E} \psi(U) \leq(p a+q b) \psi(1)=(\mathbf{E} U) \psi(1) \leq \mathbf{E} \psi(V) .
$$

To prove Proposition 16, we will need a few technical statements that we will use to replace our discrete derivatives with continuous ones. For a function $g$ on $\mathbb{R}^{n}$, let $\partial_{i} g$ denote the partial derivative with respect to the $i$ th coordinate, and let $\Delta_{i} g$ denote the discrete derivative in the $i$ th coordinate; that is,

$$
\Delta_{i} g\left(x_{1}, \ldots, x_{n}\right)=g\left(x_{1}, \ldots, x_{i}+1, \ldots, x_{n}\right)-g\left(x_{1}, \ldots, x_{n}\right) .
$$

Lemma 23. For any $g:\{0,1\}^{n} \rightarrow \mathbb{R}$, there is a unique multilinear polynomial $p\left(x_{1}, \ldots, x_{n}\right)$ that matches $g$ on $\{0,1\}^{n}$. Furthermore, for distinct $b_{1}, \ldots, b_{k} \in[n]$ and for $x_{1}, \ldots, x_{n} \in$ $\{0,1\}$,

$$
\partial_{b_{1}} \cdots \partial_{b_{k}} p\left(x_{1}, \ldots, x_{n}\right)=\Delta_{b_{1}} \cdots \Delta_{b_{k}} g\left(x_{1} \mathbf{1}\{1 \notin B\}, \ldots, x_{n} \mathbf{1}\{n \notin B\}\right) .
$$

Proof. We construct $p$ as

$$
p\left(x_{1}, \ldots, x_{n}\right)=\sum_{\left(t_{1}, \ldots, t_{n}\right) \in\{0,1\}^{n}} g\left(t_{1}, \ldots, t_{n}\right) \prod_{i=1}^{n}\left(\left(1-x_{i}\right) \mathbf{1}_{t_{i}=0}+x_{i} \mathbf{1}_{t_{i}=1}\right),
$$

which is multilinear and matches $g$ when evaluated on $\{0,1\}^{n}$. Multilinear polynomials on $n$ variables form a $2^{n}$-dimensional vector space. The map $g \mapsto p$ given above is then an injective linear map between $2^{n}$-dimensional vector spaces. Hence, it is a bijection, showing uniqueness of $p$.

For any multilinear polynomial $p$, observe that $\partial_{i} p\left(x_{1}, \ldots, x_{n}\right)$ does not depend on $x_{i}$. Thus

$$
\begin{aligned}
\partial_{i} p\left(x_{1}, \ldots, x_{n}\right) & =\int_{0}^{1} \partial_{i} p\left(x_{1}, \ldots, x_{i-1}, h, x_{i+1}, \ldots, x_{n}\right) d h \\
& =\Delta_{i} p\left(x_{1}, \ldots, x_{i-1}, 0, x_{i+1}, \ldots, x_{n}\right) .
\end{aligned}
$$

Observing that $\partial_{i}$ and $\Delta_{j}$ commute, repeated application of this shows that

$$
\partial_{b_{1}} \cdots \partial_{b_{k}} p\left(x_{1}, \ldots, x_{n}\right)=\Delta_{b_{1}} \cdots \Delta_{b_{k}} p\left(x_{1} \mathbf{1}\{1 \notin B\}, \ldots, x_{n} \mathbf{1}\{n \notin B\}\right),
$$

which proves (21).

Lemma 24. If $p$ is a multilinear polynomial, then its maximum and minimum on $[0,1]^{n}$ are attained on $\{0,1\}^{n}$.

Proof. As we mentioned, $\partial_{i} p\left(x_{1}, \ldots, x_{n}\right)$ does not depend on $x_{i}$. The function $p\left(x_{1}, \ldots, x_{n}\right)$ is therefore monotone in $x_{i}$ with the other coordinates held fixed. Thus, if $p$ achieves its maximum at $\left(x_{1}, \ldots, x_{n}\right)$, it must also achieve it either at $\left(0, x_{2}, \ldots, x_{n}\right)$ or $\left(1, x_{2}, \ldots, x_{n}\right)$. Repeating the argument it must also achieve it with $x_{2}$ set to 0 or 1 , and so on. The identical argument applies to the minimum. 
Proof of Proposition 16. Fix $(\eta, S)$ and frog paths $P_{\bullet}^{1}, \ldots, P_{\bullet}^{n}$ starting at the same vertex. We need to show that

$$
(-1)^{n} \Delta_{P_{\bullet}^{1}} \cdots \Delta_{P_{\bullet}^{n}}(\varphi \circ f)(\eta, S) \leq 0
$$

for $n=1,2$ in case (a) and for all $n \geq 1$ in case (b) Note that conditions (ii) and (iii) of the definition of icv and pgf statistics are irrelevant here, since $\varphi$ is bounded and $\varphi(\infty)$ is interpreted as $\lim _{x \rightarrow \infty} \varphi(x)$.

First, we dispense with the cases where $f$ takes an infinite value. If $f(\eta, S)=\infty$, then $f$ continues to take the value $\infty$ when additional frogs are added at the same vertex by condition (ii) of the definition. It is easy to confirm that the left hand side of (22) is then zero. For the case that $f(\eta, S)<\infty$ but $f$ takes the value $\infty$ in some term in the left hand side of (22), suppose that $P_{\bullet^{1}}^{1}, \ldots, P_{\bullet}^{n}$ is a minimal set of paths so that (22) fails. By condition (iii) there exists $P_{\bullet}^{i}$ with $f\left(\sigma_{P_{\bullet}^{i}}(\eta, S)\right)=\infty$. Take $i=n$ for simplicity. Expanding $\Delta_{P_{0}^{n}}$,

$$
\Delta_{P_{\bullet}^{1}} \cdots \Delta_{P_{\bullet}^{n}}(\varphi \circ f)(\eta, S)=\Delta_{P_{\bullet}^{1}} \cdots \Delta_{P_{\bullet}^{n-1}}(\varphi \circ f)\left(\sigma_{P_{\bullet}^{n}}(\eta, S)\right)-\Delta_{P_{\bullet}^{1}} \cdots \Delta_{P_{\bullet}^{n-1}}(\varphi \circ f)(\eta, S) .
$$

The first term on the right hand side is zero, by the argument for the case where $f(\eta, S)=\infty$. By minimality of $\left\{P_{\bullet}^{1}, \ldots, P_{\bullet}^{n}\right\}$, the second term satisfies (22) with $n$ replaced by $n-1$, which confirms (22).

For the rest of the proof, we assume that $f$ takes only finite values. For $x_{1}, \ldots, x_{n} \in\{0,1\}$, let $g\left(x_{1}, \ldots, x_{n}\right)$ be given by evaluating $f$ on the frog model $(\eta, S)$ with frogs $P_{\text {. }}^{i}$ added for each $x_{i}=1$. In the more formal notation of (5), we let $U_{x}=\left\{i \in[n]: x_{i}=1\right\}$ and define

$$
g\left(x_{1}, \ldots, x_{n}\right)=f\left(\sigma_{U_{x}}(\eta, S)\right) .
$$

Let $p$ be the multilinear polynomial matching $g$ on $\{0,1\}^{n}$. For any $x_{1}, \ldots, x_{n} \in\{0,1\}$ and $B=\left\{b_{1}, \ldots, b_{k}\right\} \subseteq[n]$, let $V=\left\{i \in[n]: x_{i}=1\right\} \backslash B$. By Lemma 23 .

$$
\begin{aligned}
\partial_{b_{1}} \cdots \partial_{b_{k}} p\left(x_{1}, \ldots, x_{n}\right) & =\Delta_{b_{1}} \cdots \Delta_{b_{k}} g\left(x_{1} \mathbf{1}\{1 \notin B\}, \ldots, x_{n} \mathbf{1}\{n \notin B\}\right) \\
& =\Delta_{P_{\bullet}^{b_{1}}} \cdots \Delta_{P_{\bullet}^{b_{k}}} f\left(\sigma_{V}(\eta, S)\right) .
\end{aligned}
$$

Thus, by our assumption on $f$,

$$
(-1)^{n} \partial_{b_{1}} \cdots \partial_{b_{k}} p\left(x_{1}, \ldots, x_{n}\right) \leq 0
$$

for $k=1,2$ in case (a) and for $k \geq 1$ in case (b) This holds for all $\left(x_{1}, \ldots, x_{n}\right) \in\{0,1\}^{n}$ and all $B \subseteq[n]$. Since partial derivatives of multilinear polynomials are also multilinear polynomials, Lemma 24 applies and shows that (23) holds for all $\left(x_{1}, \ldots, x_{n}\right) \in[0,1]^{n}$.

Now, we finish the job of transferring the problem to work exclusively with $p$ rather than $f$. By definition of $p$ in the first line and an easy induction in the second,

$$
\begin{aligned}
\Delta_{P_{\bullet}^{1}} \cdots \Delta_{P^{n}}(\varphi \circ f)(\eta, S) & =\Delta_{1} \cdots \Delta_{n}(\varphi \circ p)(0, \ldots, 0) \\
& =\int_{0}^{1} \cdots \int_{0}^{1} \partial_{1} \cdots \partial_{n}(\varphi \circ p)\left(x_{1}, \ldots, x_{n}\right) d x_{1} \cdots d x_{n} .
\end{aligned}
$$

Thus, to prove (22) it suffices to show that

$$
(-1)^{n} \partial_{1} \cdots \partial_{n}(\varphi \circ p)\left(x_{1}, \ldots, x_{n}\right) \leq 0
$$

for $n=1,2$ in case (a) and for $n \geq 1$ in case (b). This follows from the multivariate Faà di Bruno formula, the chain rule for higher derivatives (see [Har06, Proposition 1] for a 
reference). In full detail, the formula states that

$$
\partial_{1} \cdots \partial_{n}(\varphi \circ p)\left(x_{1}, \ldots, x_{n}\right)=\sum_{\pi} \varphi^{(|\pi|)}\left(p\left(x_{1}, \ldots, x_{n}\right)\right) \prod_{B \in \pi} \partial_{b_{1}} \cdots \partial_{b_{k}} p\left(x_{1}, \ldots, x_{n}\right),
$$

where the sum is over all set partitions $\pi$ of $[n]$ and $b_{1}, \ldots, b_{k}$ are the elements of $B$. Now, we fix some partition $\pi$ and determine the sign of its term in the sum. In case (b), by our assumption that $(-1)^{m} \varphi^{(m)} \leq 0$ and by (23), its sign is

$$
(-1)^{|\pi|+1} \prod_{B \in \pi}(-1)^{|B|+1}=(-1)^{|\pi|+1}(-1)^{n}(-1)^{|\pi|}=(-1)^{n+1},
$$

confirming (24). In case (a) this proof also applies, since each partition $\pi$ is into at most two blocks and each block has at most two elements.

\section{REFERENCES}

Akh65. N. I. Akhiezer, The classical moment problem and some related questions in analysis, translated by N. Kemmer, Hafner Publishing Co., New York, 1965. MR 0184042

AMP02a. O. S. M. Alves, F. P. Machado, and S. Yu. Popov, The shape theorem for the frog model, Ann. Appl. Probab. 12 (2002), no. 2, 533-546. MR 1910638 (2003c:60159)

AMP02b. Oswaldo Alves, Fabio Machado, and Serguei Popov, Phase transition for the frog model, Electron. J. Probab. 7 (2002), no. 16, 1-21.

AMPR01. O. S. M. Alves, F. P. Machado, S. Yu. Popov, and K. Ravishankar, The shape theorem for the frog model with random initial configuration, Markov Process. Related Fields 7 (2001), no. 4, 525-539. MR 1893139 (2003f:60171)

DP14. Christian Döbler and Lorenz Pfeifroth, Recurrence for the frog model with drift on $\mathbb{Z}^{d}$, Electron. Commun. Probab. 19 (2014), no. 79, 13. MR 3283610

FMS04. L. R. Fontes, F. P. Machado, and A. Sarkar, The critical probability for the frog model is not a monotonic function of the graph, J. Appl. Probab. 41 (2004), no. 1, 292-298. MR 2036292 (2004m:60221)

GS09. Nina Gantert and Philipp Schmidt, Recurrence for the frog model with drift on $\mathbb{Z}$, Markov Process. Related Fields 15 (2009), no. 1, 51-58. MR 2509423 (2010g:60170)

Har06. Michael Hardy, Combinatorics of partial derivatives, Electron. J. Combin. 13 (2006), no. 1, Research Paper 1, 13 pp. (electronic). MR 2200529

HJJ16a. Christopher Hoffman, Tobias Johnson, and Matthew Junge, From transience to recurrence with Poisson tree frogs, Ann. Appl. Probab. 26 (2016), no. 3, 1620-1635. MR 3513600

HJJ16b. _ Recurrence and transience for the frog model on trees, to appear in the Annals of Probability, available at arXiv:1404.6238, 2016.

JJ16. Tobias Johnson and Matthew Junge, The critical density for the frog model is the degree of the tree, Electron. Commun. Probab. 21 (2016), no. 82, 12. MR 3580451

KZ16. Elena Kosygina and Martin P. W. Zerner, A zero-one law for recurrence and transience of frog processes, to appear in Probability Theory and Related Fields, available at arXiv:1508.01953, 2016.

LMP05. Élcio Lebensztayn, Fábio P. Machado, and Serguei Popov, An improved upper bound for the critical probability of the frog model on homogeneous trees, J. Stat. Phys. 119 (2005), no. 1-2, 331-345. MR 2144514 (2006b:82068)

LT14. Junghoon Lee and Cihan Tepedelenlioğlu, Stochastic ordering of interference in large-scale wireless networks, IEEE Trans. Signal Process. 62 (2014), no. 3, 729-740. MR 3160309

Mar02. R. Marchand, Strict inequalities for the time constant in first passage percolation, Ann. Appl. Probab. 12 (2002), no. 3, 1001-1038. MR 1925450

Pop01. Serguei Yu. Popov, Frogs in random environment, J. Statist. Phys. 102 (2001), no. 1-2, 191-201. MR 1819703 (2002a:82064)

RS04. Alejandro F. Ramírez and Vladas Sidoravicius, Asymptotic behavior of a stochastic combustion growth process, J. Eur. Math. Soc. (JEMS) 6 (2004), no. 3, 293-334. MR 2060478 (2005e:60234)

SS07. Moshe Shaked and J. George Shanthikumar, Stochastic orders, Springer Series in Statistics, Springer, New York, 2007. MR 2265633 (2008g:60005) 
Sta12. Richard P. Stanley, Enumerative combinatorics. Volume 1, second ed., Cambridge Studies in Advanced Mathematics, vol. 49, Cambridge University Press, Cambridge, 2012. MR 2868112

TRZ11. Cihan Tepedelenlioğlu, Adithya Rajan, and Yuan Zhang, Applications of stochastic ordering to wireless communications, no. 12, 4249-4257.

TW99. András Telcs and Nicholas C. Wormald, Branching and tree indexed random walks on fractals, J. Appl. Probab. 36 (1999), no. 4, 999-1011. MR 1742145 (2001m:60199)

vdBK93. J. van den Berg and H. Kesten, Inequalities for the time constant in first-passage percolation, Ann. Appl. Probab. 3 (1993), no. 1, 56-80. MR 1202515

Zer98. Martin P. W. Zerner, Directional decay of the Green's function for a random nonnegative potential on $\mathbf{Z}^{d}$, Ann. Appl. Probab. 8 (1998), no. 1, 246-280. MR 1620370

Department of Mathematics, New York University

E-mail address: tobias.johnson@nyu.edu

Department of Mathematics, Duke University

E-mail address: jungem@math.duke.edu 